$G-E$ 


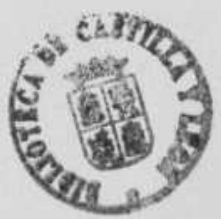

$R .76710$ 


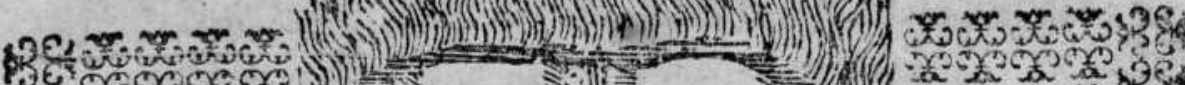

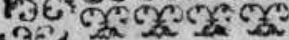

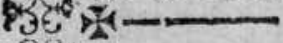

अध一彑

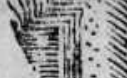

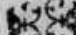

36

और

s.

s.

PJE

sis

sis

836

ats

sis

6 सेत्र

हु,

तु

s.

急政

$\sin 5$
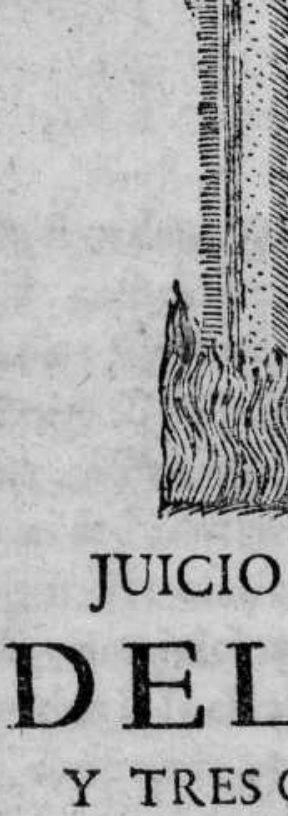

sis

PE

s. 3 द्रुक

635

OUE

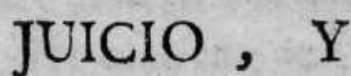

$\mathrm{Y}$

PRONOSTICO

23

Orizonte Efpañol el dia dos de Noviembre de efte
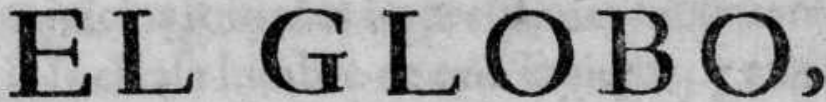

Y TRES COLUMNAS DE FUEGO,

केष्ध

\$त्रत

रुद्ध

STS POR DON DIEGO DE TORRES VILLARROEL,

ह2.

उद्ध

किष्द

Catbedratico de Prima de Matbematicas en la

Univerfidad de Salamanca.

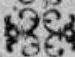

+3द्ध

अंडृ año de 1730 . y unas Preparaciones Medicinales muy dulces, para librarfe de la malicia de fus vapores, y humos.

30

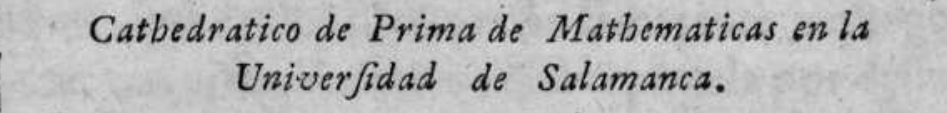

C(JN LICENCIA: En Madrid, por Antonio Marin. Vendere en fu Cafa, en la Calle de fejus Maria, frente la Porteria de la Merced Calxada; y en la Libreria de fuan de Moya, frente S. Felipe el Real.

936

की

के हैं

38

?3

शुद

क्षेक

किज्ञ

अंड़

के है

कुष

के 38

क्रिख

कर्ड

कर है

अहैं

और्जि

उद्ध

संद्ध

s.

Es

Eis

क्जित्र

केंद

की द्धि

क्रिद्ध

होंड़ी

अध

Sig द छब 8र 94 VG 


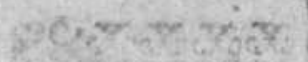

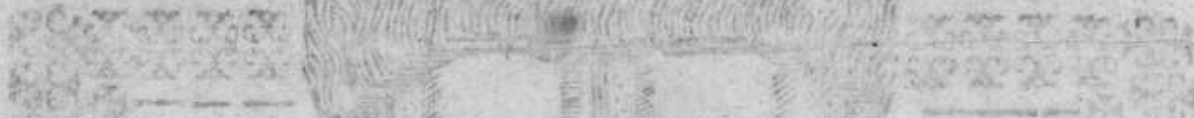
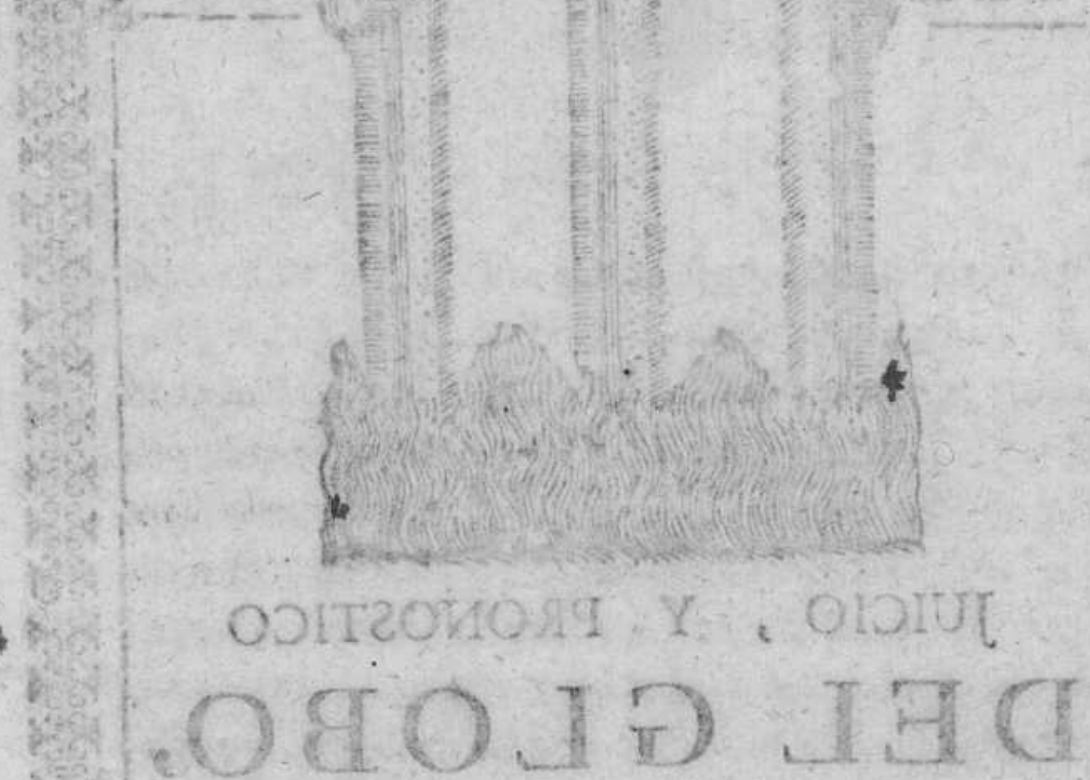

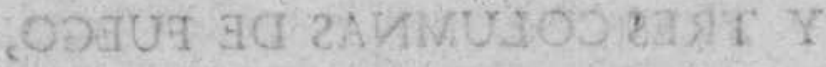

ige

tosis

5.325

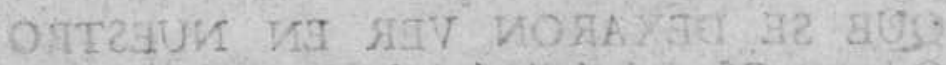

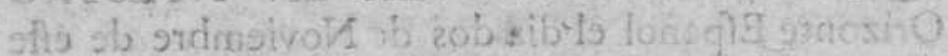

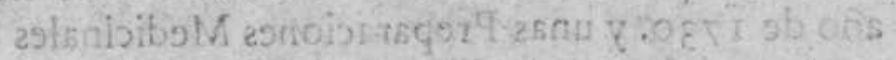

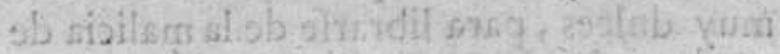

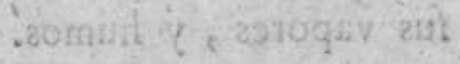

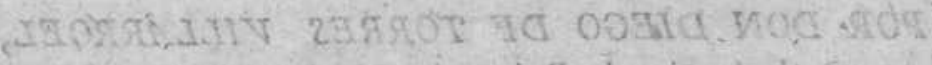

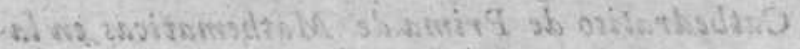

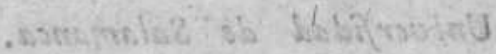

i

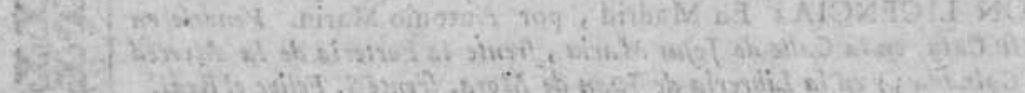
Yinge

s. 
APROBACION DE DON ZENON GUERAO Aznar, Profeffor de Aftronomia , y Medico en efta Corte.

\section{P. S.}

E orden, y comission de V.A. he vifto con atencion prolixa, y cuidadofa, el Dapel, inticulado: Re/puefta de Don Diego de Torres y Villarroel d) fu amigo Don fuan Ventura, Jobre la aparicion del Phet: nomeno, ò Promontorio de luces, que fe dexò vèr en el ayre el dia 2. de Noviembre de efte año de 1730 . Examinado en rigor efte eferito, tan folo hallo, que comprehende unos juicios fobre las imprefsiones fublunares; formados à la efcafa lumbre de una incierta, $y$ engañofa congetura, y deducidos de la deftemplanza del ambiente, ocafionada ( fegun fe fofpecha) del fupe-rior Phenomeno que ha aparecido en varios lugares de nueftro Orizonte. Es cierto, que fi en la ignorancia de los Pueblos encontraren eftos prognofticos tan difpuefta, como fiempre, la credulidad, no ferà porque fu Autor los ha propuefto con aquella affeveracion, que en los Efcritos de femejante cathegoria, con tan jufto derecho, eftà repurada por delinquente. A la verdad, defpues de confeffarle à Don Diego de Torres las fales, y pimientas con que fuer le fazonar fus Papeles, no puede negarfele tampoco el difcreto, virtuofo, y prudente defengaño; el in- 
gento, y admirable candor con que efcrive; y la poca fee que fe le debe à fus predicciones. Todos los Aurores que componen Pronofticos, fe farisfacen, con eftampar en el Dios fobre todo el caracter de fu religiofa deferencia ; pero. Don Diego, no contento con efta publica profefsion de fu humildad, y fujecion, teftificalfuerade ella en todás las obras de efte jecion , y aùnen muchas que no do fon, la incertidutubre à que eftàn fujectas fus congeturas, hafta perfuadir la vanidad de rodos fus oraculos, y la folemnidad de fus errotes : para dàr credito à los demàs, bafta fer ligero : para dàr credito à efte Autor $s$ ferequiere ferobottinadol En efta Carta contefta to mila amo, con placifible finceridad ; bieri, que conforme à los principios mas comunes Phifico-Aftronomicos, philofopha fobrezel dicho Phenomeno, reffriẹido à la conftivucion contaminada del ayre los futares contingentes, y foneflos efectos que predicos por tanto, no tienen que concebit temor los lectores, pues el mifmo Autor refponde con franqueza lo que folicivarèeadoerrar en los numeros de la decizma que ferfigue.

\section{DE C I M A.}

Del ayre que no es propicio, arguye Torres: fatal

de todo el Reyno animal

el formidable perjuicio.

No tengas miedo à fu juicio, 
lector, que al tuyo defayre, pues refponde con donayre, aùn quando tu no lo a puras, que effas mifmas congeturas vàn fundadás en el ayre.

En fin, Señor, en efta Obra folo hallo un irreprehenfible entretenimiento, fin advertir en toda ella linea alguna que contradiga à las Ordenanzas Eclefiafticas, ni Civiles, por lo que puede V.A. conceder à fu Autor el permiffo que pretende para eftamparla. Efte es mi parecer, teniendo fiempre la debida veneracion al mejor dictamen. Madrid, y. mi Pofada 2 9. de Noviembre de 1730.

D. Zenon Guerao Aznar. 


\section{LICENCIA DEL CONSEJO.}

On Miguèl Fernandez Munilla; Secretario del Rey nueftro Señor, fu Efcrivano de Camara mas antiguo, y de Govierno del Confejo: Certifico, que por los Señores de èl $f e$ ha concedido licencia para poder imprimir el Papel, inticulado: Refpuefta de Don Diego de Torres y Villarroel d $\int u$ amigo Don fuan Ventura, fobre la aparicion del Pbenomeno, ò Promontorio de luces, que fe dexò bèr en el ayre el dia 2. de Noviembre de efte año de 1730. compuefto por Don Diego de Torres, Ca: redratico de Prima de Mathematicas en la Univet: fidad de Salansanca, como mas largamente confta de fu original. Madrid, y Diciembre r. de 1730.

D. Miguel Fernandez Munilla: 
RESPUESTA DE DON DIEGO de Torres à fu amigo Don fuan Ventura, Sobre la aparicion del Pbenomeno, ò Promontorio de luces, que fe dexò vèr en el ayre el dia dos de Noviembre de efte año de mil Setecientos $y$ treinta.

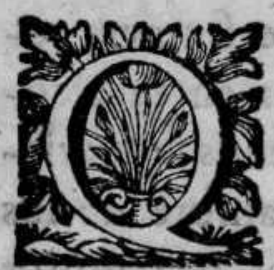

UANDO eftaba yo fufriendo las inpertinentes congoxas de una melancolia, (huefped tan pegajofo, y remolòn, que ni los defacatos de la Medicina, ni los defayres de mi genio, han podido defecharlo de mis hypocondrios) recibì la de V.md. feñor Don Juan Ventura, y penfando hallar en las feltividades de fu nota, alguna convalecencia de mis manìas, di de ojos (Dios nos libre) en el horrorofo dibujo de un peregrino Promontorio deluces, y nubarroncs, que fe fixò en una de las esferas eminentes à nofotros, el dia dos de Noviembre de efte ańo; y lo peor es, que me confulta V.md. y me quiere examinar en fu naturaleza, como fi yo fueffe inquilino del Ether, ò compańero de alguno de los Satelites de Jupiter. Mucho fiento que fe ayan perdido las perfuafiones de mi pluma, y las confianzas de mi conyerfacion con V.md. fobre lo oculto de eftas materias. Por cierto, que go creìa que V.md. avia buelto à recoger el juicio que fe le avia efcapado por el agugero de 
las predicciones, y trifte de mi ! hallo que no fe contenta V.md. con fer loco, y que lo fean fus hijos, fino que quiere defnudarme a mi del débil tegumento de la razon con que fe arropan mis pobres cafcos. Acuerdome de un hombre de quien hace memoria Juan Barclayo, y aun dice que le tratô en Mergania. Elte, pues, era de opinion tan temo$\mathrm{fa}$, y tai confultor de las eftellas, que fin aver repaffado primero tos Aftros, no entraria en la alco. ba àrafcarfe con fu nifuger, aunque Venus le huviora introducido en fus rinones un enxambre de fins pulgas; y por mas que menudeaffen los terremoros del pais baxo, la vez que Mercurio eftaba de mal humor, ò el Scor pion echaba un por vida, ù otro qualquiera Afterifmo la juraba en el Cielo, dormia folo: otras veces, quando en el campanario del Cielo tocaban à engendrat, fembraba en fu Haza; y lo que fucediò à rodas eftas obfervaciones, fue, que le nacieron algunos hijos, pero todos calvos de juicio, como fu padre. No quiera V. md. parecerfe à efte loco; dexefe de conjeturas necias: G lllueve, recojafe en fu cafa: fi hace Sol, gocelo: fi aparecen Cometas, dexelas V. md. pues por muchia mortandad que amenacen, à V.mond. no le ha de faltar una hora para morirfe.

Que à ninguna efpecie de Oraculo fe ha de coufultar, he intentado perfuadir à V. md. varias veces, y aora nuevamente me armo con efte argu- 
miento. O fe predicen fuceffos lamentables, ò profperos. Si profperos, los cree V.md. y le engañan: eftà condenado à las galeras de una ef peranza, adonde ay Comitres mas crudos que los Genovefes. Llamò uno à la efperanza martyrio cruel, en que merece el mas indigno el cielo de la poffefsion. Què diria de la efperanza vana? de cuyas galeras, mas facala defefperacion para las fogas, que el defengaño para los hiermos. Si los fuceffos que le predicen à V.md. fiendo profperos los cree, y es cierto que han de venir, ( dado que en la efperanza empieze à vivir el gufto ) fe defloran antes de la poffefsion, de fuerte, que venida efta, ferà faftidio lo que avia de fer gozo; y comiendofe las peras verdes, es defigual el deleyre que fe anticipa V.md. al que pierde por anticiparfe. Es la efperanza una furia tan maligna, que pone al que efpera lexos del bien, para que con la impaciencia del defeo fe atormente el animo; y lo acerca tambien al bien, para que al primer inftante de poffersion fe empalague el gufto. Pues quien no defefpera con tal efperanza, aun fiendo del bien ? Si à V.md. le anuncian fortunas contrarias, los cree, y mienten; fe queda temiendo el mal que no ha de venir, no aviendo peor mal que el temor: con que fiendo para con V.md. piadofa la fuerte, es cruel contra sì mifmo ; y lo que debiera temer, es cl temor. Finalmente, fi predicen fuceffos contrarios, y no 


\section{4}

mienten, V.md. fe negocia de antemaño el temor, $y$ antes del tormento es atormentado, y fe pone à la vanda del que le perfigue; y afsi, no fé quexe de la fortuna que le dilata el martyrio, y V.md. fe anticipa à entregarfe à los verdugos; y quizà quando viene el dańo, no hallarà en quien executar fu eftrago. Luego, para què fin quiere $V$.md. confultar Oraculos, ni Eftrellas, fi qualquiera refpuefta à cerca del fururo fuceffo, no es perniciofa, fea falàz, ò verdadera, adverfa, ò profpera?

Acuerdome tambien, que en uno de los parrafos de fu carta, (que no feńalo qual es, por no tenerla à mano ) dice, que prefume una eficàz copia de enfermedades, y me pide remedios para huir de los contagios del Phenomeno. Señor Don Juan, para mi los he pedido yo al mayor numero de los Doctores que tratan en remendar cuerpos, y ninguno me los puede dàr. Si yo fupieffe Medicina contră la mala condicion de los años, huviera librado à mi padre (que Dios aya) de la muerte, ò me la preftarian, fi la conocieffen, los Medicos que le vifitaron, y yo me defcartàra de un rehumatifmo, que hà dos ańos que me eftà mordiendo la reñonada. Señor mio, lo que yo puedo hacer, (procediendo con imitacion de otros ) es feñalarle à V.md.quatro vegetables, ò veinte compoficiones de los fugetos que crian, y mantienen los tres Reynos Mineral, Ani$\mathrm{mal}$, y Vegetable, ò el puńal de una lanceta, ò las 
brujas de vidrio, ù otros embelecos que canonizan los Phyficos en fus practicas; pero ni ellos, ni yo podemos affegurar à V. md. de fu virtud, ni de la fanidad que bufca. Acabe V.md. de creer que es mortal, y que defde que nacemos eftamos moribundos, y que no ay remedio para librarnos de efte schaque : fufra V.md. y no pienfe en locuras. Oyga V.md. un cuentecillo, que puede fer que con fis exemplo fe apee de fus ignorancias.

Vivia en Salamanca una vieja, à expenfas de muchos tontos, que dexandola en fu cienda el metal, la compraban la adivinacion : ( que con femejantes mercancias fe mantiene la mitad de el mundo : todo èl es cafa de locos, y cada Reyno, ò Provincia un apofento de la cafa; y fi un dia amanecieffe cuerdo, y perfeveraffe un mes con feffo, yo sè que murieran a millones los Mercaderes de muchas haberìs, y que pudiera el hambre apoftar à matar con las albardas, y los Doctores) la vieja, pues, vendia refpueftas, y era fu cafa la botilleria univerfad, donde bebia todo ignorante curiolo. Sucediò que en la de un hombre acomodado hurtaron una alhaja de grande cftima : advirtiòlo un criado mayor, à cuya fidelidad, y vigilancia tenia confiada el feñor toda fu riqueza, el qual con otro compañero, determinaron hacerle un voto à la vieja, para que con el indice de fu fabiduria, les encaminàra al gavilàn que hizo la prefa. Ibà à la cafa del Oraculo con fu compañero, 


\section{6}

y à cada pafro que daba para llegar à ella, le parecia que agarrabaalladron, y que le reftituia fu prenda; pero bien prefto el fuceflo les defvaneciò la confianza. Era bien de mañana, y llegaron los dos à las puertas dela vieja, al tiempo que abriendolas, hallo el umbral lleno de mocos de trafero con romadizo, tal que fe defpediria de èl, mas velóz quo una flecha, qualquiera que no fueffe amigo de tomar colas de Botica. No fe fupo quien de los vecinos la noche antes convirtiò el umbral en pańuelo de fu hediondo catarro, por abaharle las barbas à la maldita vieja. Luego que à efta fe la enlodaron los ojos, y recibio el guijarrazo en las narizes, convocò en fu venganza un efquadròn de juramentos, y maldiciones, y al cabo dixo: Por la Soberana Deydad de Apolo, que fi fupiera quien es, el que favorecido de las tinieblas de la noche (que es la carantula de los picaros vergonzantes) ordenò que pagaffen mis umbrales pechos de muladar; por la Soberana Deydad de Apolo, buelvo à decir, que le echaria en fu holla el culantro que fembrò à mi puerta. Aun no acabò de leer la vieja fu carta de excomunion, quando el que iba à confultarla fe defmontò de la necia confianza que lo fobftenia ; fino es que cayò la burra, y el coftal, y mirando con vehemencia al compañero, le dixo: Hombre, què vamos à hacer ? cl defengaño nos fale à recibir à las puertas de la profecia: antes que huvieramos oido lo que acabamos de oir, eramos mulos novicios en folicitar ref- 
pueftas de una vieja engatufadora, y caduca, figuiendo à la gran beftia del vulgo; peroli defpues profeguimos con nueftro intento, llegarà el dia de li profefsion : efto es, por bufcar el huevo de oro, matar la gallina, y perderlo todo. Por ventura, fabrà cfta vieja con determinacion, quien fue el que facò la preciofa alhaja de nueftra cafa, quando no ha podido faber quien metiò el eftiercol en la fuya ? dixo, y fe bolvieron ambos defengañados. Aplique V.md. el cuento, y advierta, como podrè yo determinarle remedios, ni preparaciones para fu falud, quando no puedo yo facudir las chifpas de una fluxion que me las eftà jurando de muerte en las Eftaciones de Primavera, y Otońo. No obftante, pues eftamos obligados à poner los medios humanos, y feguir las confideraciones que eftuvieren de nueftra parte, para lograr menos motin en los humores, he de fervir à V. md. en todo lo que me manda; y con fencillèz, y verdad, dirè lo que fentì el dia que apareciò el efcandalofo Phenomeno que oy tiene affuftados los animos, y en contemplacion los difcurfos; y al fin de mi explicacion, recitarè un breve methodo para prefervarfe de los daños que eftà fignificando en los cuerpos. 


\section{8 \\ GENERACION DEL Globo, ò Phenomeno de luz.}

L experiencia acreditada entre los Philofophos contemplativos naturales, efperar malos fuceffos en la tierra, quando aparecen femejantes fir: guras, y tan eftrañas vifiones en el ayre, ò en el fuego; pues nunca pueden paffar de eftas dos regiones tan monftruofos afpectos. Es tan natural la generacion de eftas imprefsiones, como loes la de la lla: via, el granizo, la nieve, los truenos, y relampagos, nubes, y otros Phenomenos, que por comnnes pierden nueftra contemplacion, $y$ eftudio. $\mathbf{L a}$ congregacion de los Aftros, con cuya variedad fe aumenta, y diftingue la hermofura del cuerpo Celefte, no defpierta, ni convoca los animos; y hemos menefter para mirar al Cielo, aguardar à que padezca alguna obfcuridad el Sol, ò algun trabajo la Luna, ù otra novedad en alguna de las esferas fuperiores; y folo quando ay efta, dàn gritos las gentes, y Ciudades, fin quedar angulo (por remoto que $f e a$ ) que no tema algun peligro: como fi para acabar la vida (que es el mayor mal que fe nos propone) fueffen neceffarios mas fignos, que el que cada individuo conferva en fu organizacion. Digo, pues, que la continuz tarèz de los movimientos de 
los cuerpos celeftiales, que con fus aceeffos, y receffos al Orbe terraqueo, yà le ocupan defus influxos, $y$ virtudes, yaje forben los proprios que èl contiene en-ambos Elementos de tierra, y agua, fon la caum fa de elevar à la region del ayre eftas materias, ò fuccos de ambos elementos, y en ella fon detenidos, eongregados, y encendidos, yà por el Sol, ò por la agitacion de unas particulas con otras. Unas veces fe deshacen brevemente, y efta es la llubia; otras fe quaxan, y efte es granizo; otras veces fe quedan mas crudos, y caen en copos, y efta es nieve; otras fe unen, y cuecen tanto, que encerrada en la nube la materia mas fulfurea vituminofa, y efpirituofa, pelea por falir, $y$ hace el ruido que llaman trueno; y à la llama que exhala al falir, relampago; y à la materia sòlida contenida en la nube, rayo, ò centella; y no tienen otro origen, ni otra generacion eftos Meteoros : $y$ todos los vifibles fe forman, coagulan , y difponen desuna mifma materia, que fon los vapores, humos, y alientos del agua, $y$ la tierra, elevados por el fuego del Sol, y la virtud de los demàs cuerpos celeftes, à la region fuprema, ò media del ayre; $y$ fegun el calor, humedad, ò fequedad de aquella esfera, fe deshacen, ilefvanecen, ò quaxan: y alli, fegun la unionile las materias, y fu temperamento, forman laśfigoras tan raras que hemos vifto. Pues fin paffar à oura confideracion que la de las nubes, à unas las veèmos redontas, otras quadra- 


\section{0}

das, otras en ovalo, otras largas; y lo mifmo fuce? de en lo colorido de ellas, pues yà fon azules, yà verdes, yà negras, y de otros diverfós, y aùn imperceptibles colores : y la caufa de formar elta variedad de figuras, y colores, no es otra, que lo grueffo, pingue, tènue, ò dèbil de la materia, unida por la virtud del ayre mas à una parte, que à otra, y la varia radiacion del Sol, ù de ocro cuerpo Planetario; porque fegun el lado por donde es mayor la iluminacion, aparecen mas claras, dexando los demàs lados de la figura mas, ò menos obfcuros, ò iluminados, fegun la difpoficion del cuerpo opaco ; y la luz, como acà la vèmos en un candil, y nueftros cuerpos; pues por el lado que dà la luz, eftàn mas claros que por el otro adonde no la reciben. Para hacer eftos difcurfos, ni es menefter aver curfado las Univerfidades, ni fer Carhedraticos de Aftrologia, que la razon natural lo parla al mas rudo.

Los mas de los años fuelen aparecer en la ef: fera del ayre (efpecialmente en los fines de Ootubre, y Noviembre, y mas fi hace en ellos algun calor) figuras, globos, y vifiones de luz de efta nay turaleza; pero no fiendo de la quantidad defmefurada que la que oy nos hormoriza, no fe hace cafo de ellas; y quando mas, migtecen la memoria de los Arrieros, y caminantes en los mefones, ò los Nauticos en el Valo; y entre efta gente le queda la noticia, que à los vecinos de las poblaciones cor- 
refanas, y politicas, cômo duermen mas, y madrugan menos, no les defacamoda la curiofidad de eftas obfervaciones. El dia diez y nueve de Octubre del año de mil fetecientos y veinte $y$ feis, apareciò en nueftros Orizontes otro Globo de luz, de la mifma naturaleza que el prefente; y me acuerdo, que defcrivi entonces fus influxos, y caufas; y pues alli determinè mi opinion, no quiero repecir thas doctrinas. Defde que viò nueftro Orizonte aquella pe-j regrina, y prodigiofa luz, no hemos logrado las determinadas templanzas de las Eftaciones, ni los cuetpos han podido facudirfe de las malignas qualidades que recibieron de fus foplos; pues ( como di$x e$ entonces) fe figuieron varias, y eftrañas enfermedades en los racionales, y brutos; y la cofecha de vegetables, ha fido pobre, debil, y enferma en todas las poblaciones de nueftra Efpaña : y defde aquella eftupenda imprefsion, hà que vivimos oprimidos todos los fublunares, yà embueltos en nieve, como fucediò el año de 1729 . yà en frios deftemplados irregulares, como padecimos en la Primavera, y en lo mas del Eftio del ańo de $\mathbf{1 7 3 0}$. figuiendofe à eftas irregularidades del tiempo, la miferia, enfermedad, y mal acondicionado temperamento de los cuerpos de todas efpecies; ni podièmos convalecer tan aprifa, fi no clamamos à Dios, que es verdadera vida, $y$ falud.

Muy varia ha fido la noticia de la aparicion de 
efte Promontorio de fuego $\bar{a}$ y todos defconvienen en la figura: de efto no me admiro, ni digo que nos engañen los que lo han affegurado; porque es cierto, que fegun es la fituacion del lugar donde fe obferva, afsi aparece tambien vario el cuerpo: $y$ defde un lugar fe defcubre mas que de otro; $y$ afsi, unos moradores vèn los eclypfes, y otros no: $y$ aun el Sol defde unos lugares parece redondo, y defde otros ovalado. Del Reyno de Navarra, efcrive Don Carlos Arslegui, Cura de Equifoayn, que le obfervò camino de Monreal, en el monte de Alaiz de di, cha Villa, con orros amigos, $y$ algunos paftores, à los quales à las dos horas defpues de la prima noche del dia nueve de Oetubre de efte ańo, los affuftò una claridad quafí igual à la del Sol, con cuya peregrina luz regiftraban con toda diftincion los montes, he-; redades, y Pueblos circunvecinos. Dos horas affer: guran eftos verdaderos obfervadores, que fue la duracion de la luz; y al finde ellas, defcubrieron un nubarron, ò globo monftruofo de fuego àcia la parse del Orgiente, y efte durò una hora, exhalandofe la mayor parte de fus humos al fitio del Poniente. En el mifmo lugar donde fe formò el Promontorio de fuego, aparecieron tres Columuas grandes del mifmo color, y encendimiento que el Globor: la columna del medio, fe defvaneciò en el efpacio de media hora; $y$ las otras duraron hafta las quatro y media de la mańana, que faliồ la Luna. De Andalucia 
me embiaron dibujadas eftas mifmas figuras, que obfervò otro curiofo à las mifmas horas que hemos dicho fe aparéciò en la Navarra. Algunas orras perfonas de verdad, me han affegurado aver vifto en otras noches varias vifiones de fuego en la region del ayte : yo folo dirè à V.md. lo que he vifto, y lo que fiento de efta nueva, y fatal aparicion.

El punto fixo de la generacion, y defcubrimiento de efte Phenomeno, no pudo ferme notorio, y efto mifno fucede comunmente en todos quantos hafta oy fe han vifto; pues por la mayor parte, fu principio es incognito, y folo puede conocerfe quando alguna noche ferena eftamos mirando al Cielo, y de repente aparece la vifion: entonces fe obferva la altura que tiene fobre el Orizonte, y la de dos, ò tres eftrellas de las conocidas, en longitud, y latitud; y afsi fe examina la hora de fu principio, y fe miden los grados de diftancia defde ellas al Phenomeno, para colocarle en fu lugar verdadero. Lo que regularmente fucede en eftos cafos, es, que paffados uno, dos, ò mas dias, fe advierte, y nota: por lo qual, fiempre ignoramos el principio, dia, y hora de fu aparicion; y por configuiente, no fe fabe fi fe encendiò en nueftro Emisferio fuperior, ò en el inferior ; fi fue de dia, ù de noche: por cuya caufa, es comun doctrina de los Aftrologos, recurrir à las radiaciones, ò afpectus que pudieron iluminarlo, ò encenderlo, viendo en la conjuncion, ù opoficion prevencional 
à la aparicion del Globo, ò Phenomeno, toda la configuracion de las Eftrellas errantes; y fegun la varia pofitura de ellas en el Zodiaco, y lugar de la pri-; mera obfervacion, fe viene al conocimiento de fu principio, generacion, y lugar donde empezò à lucir.

El dia dos de Noviembre à las r r. y 30. minutos de la noche, obfervè yo defde efte Orizonte Salmantino, en la parte meridiana, entre el figno del Cancer, y el Leon, y parte de la conftelacion de Virgo, in eftupendo Globo de fuego, tan fobervio como el edificio mas famptuofo de la Corte : eftaban de colaterales à efte Promontorio dos rafagas, ò coJumnas, que à la vifta, me pareciò que fubian, y baz xaban, $y$ adquirian con el movimiento mayor luz; y claridad. El color de las columnas era vario, poriue yà aparecia verde, yà encarnado, y en la cima fus perior del Globo, fe regiftraban dos grandes llamas ceruleas, y del color del fuego. La tieria, me pareciò que vomitaba al infierno que tiene en fus entrañas, fegun las arqueadas de lumbre que defpedia à las dos de la mañana, que à efta hora le encendiò todo el Globo, $y$ fe unieron las columnas; $y$ fu duracion fue hafta las quatro y media. Efto es lo que yo he vilto: oyga V.md. lo que difcurro.

No difputo, ni ignoro, que efta luz fea algun Embaxador Celeftial, que embiado de Dios, venga à perfuadir al Genero Humano fu ira , y los juftos 
motivos de fu indignacion; porque de eftas feñales, fe firve fu providencia quando es fuvoluntad: $y$ affentado primeramente, que puede fer figno fobrenatural, cada pobre difponga fu alma, por fi acafo le llama Dios à dàr la ultima cuenta. En la naturale$z a$, fe defcubren caufas baftantes para tales, y mas crecidas monfruofas vifiones; y al'si, no nos agarrèmos de los milagros, quando ella apunta la caufa de efte prodigio; porque es floxedad del entendimiento, penfar que para fabricar eftas figuras, es neceflaria la potencia abfolura de Dios, quando bafta folo la ordinaria; y de elta es apoderada la naturaleza. Yà fe acuerda V.md. de las rigurofas nieves, y rebeldes heladas del Enero de I 72 9. yà fabe V.md. por la Filofofia, que la materia de la nieve, la lluvia, y los demàs habitadores que fe crian, y forman en la esfera del ayre, es eftefucco, atomos, y cuerpecillos de la tierra, que en virtud del calor del Sol, los arroja ella, como eftraños, defu centro; y fabe V.md. que fon fulfureos, vituminofos, falinos, y nitrofos, y de otras qualidades, que aùn fe ignoran. La nieve, es cierto que baxa coagulada à la tierra, por la virtud de la nitro, ò fal que la quaxa, y no pudo el ayre diffolverlo, por la poca difpoficion que goza de calor en el Invierno en fu esfera. Pues efte nitro, ò $f a l, y$ eftas particulas de la nieve, quedaron en la fuperficie, y media region de la tierra todo el ańo de 1729. y en el Julio, y Agofto del mifmo año que fe eleva- 


\section{6}

ron à la region del ayre, no las pudo cocer, difsipar, ni refolver en llavias, truenos, granizos, ò relampagos, por la corta actividad de fu calor. Quedò, pues, con efta eftrańa malicia impuro, agoviado, y rullido el ayre, como lo experimentamos ( $\mathrm{fi}$ V.md.hace memoria) en aquellos mefes, que no fe moviò un foplo de viento; y por efta quietud fe unieron, y fe condenfaron con fuerza mayor eftas particulas, $y$ atomos fulfureos: y como bebimos en la impuridad del ayre eftos azufres, padecieron nueftros cuerpos la epidemia de tercianas del ańo paflado, cuyo fermento era una exalcacion de la colera, movida por eftos halicos, humos, y mala condicion del ayre ; y por elta razon, los mas tercianarios padecieron ol decubito à los reñones, al eftomago, è inteftinos; y à muchos (como yo lo vì) fe les dañó el pulmòn, y murieron. Los vegetables, y animales en dicha Efracion, fe criaron en fuma debilidad : los frutos, fin aquella hecmofura, humedad, y robultèz, crecieron aridos, dèbiles, y mal complexionados. Los ganados, no fe vieron libres de la rońa, enteco, viruela, y orras enfermedades. Todo efto lo viò V.md. con que hafta aqui vamos feguros.

Quedòfe el ayre lleno de eftas impurezas, grueffo, cargado, yenfermo; y en los mefes del Octubre, que efperabamos que fe defahogaffe un poco confrequentes lluvias, ( $y$ aunque cayo alguna à la tierra, no fue tan copiofa, que pudiefre facudirfe de 
fu hydropesiá) en el Inviernô bolviò à hincharfe de las nieblas, y de las otras parriculas, que continuamente eftà chupando del Sol; y los demàs cuerpos Celeftiales condenfaronfe mas; y à aquel fulfur, y nitro de la nieve del año de 1729 . lo encerraton, y unieron con mas eftrechèz. No ha logrado el ayre ocafion hafta oy de defocuparfe; porque en el Verano, y parte del Eftio de efte año, ha fido mas el frio que el calor: ( fiendo la caufa de efte, la claufu-: ra, y union de las particulas fulfureas, ix nittofas) como eftas no pudieron falir à deshaceto las pattes flemofas que las aprifionaron en el Invierno, de aqui fue la deftemplanza fria del Verano: con que no aviendo podido defembarazarfe de efta opilacion, como todos hemos vifto, ciertamente podèmos affegurar, que eftuvo la esfera del ayre gravada de eftas particulas malignas. En el Agofto, y Septiembre de efte año, fue moderado el calor, y el viento, generalmente $\mathrm{fe}$ moviò en todas partes; pues con efta circulacion, y el moderado fuego de eftos mefes, fe fueron fecandot, y moviendo las partes frias, y flein ofas de las nubes, dando tranfito à los atomos, ò particulas nitrofas, fulfureas, que eftaban encerradas, y obftruidas por eftas otras, cayendo à la tierra algunas de las partes flemofas en lluvias benignas, como lo experimentamos en el Sepriembre; y refolviendofe otras, ò incrafandofe, 
quedò el ayre mas defahogado, y en èl preparadas (por el movimiento, y por la aufencia de las pars tes aqueas) las partes efpiritofas, y falinas.

Note V.md. aora, y vamos juntando principios. La tierra, defde las rigurolas heladas del Enero, y Febrero de 1729 . quedò fembrada del fulds fur, fales, y nitros de la nieve: eftuvo conftipada hafta el mes de Junio de dicho ańo: en la Eftacion del Eltio fe elevaron eftas materias del ayre: alli no fe pudieron refolver, ni difsipar, por la poca difpolicion del calor, y la dèbilidad de los influxos celeftiales, antes bien fe condenfaron, y oprimieron. En el año de 1730 . tampoco hemos vifto el defahogo del ayre de eftas partes efpiritolos; porque ni en relampagos, truenos, ni granizos, han caido à la cierra las porciones que correlpondian à dos mefes de nieve, $y$ hielos continuados, con que alli eftuvieron detenidas hafta los principios del Octubre de efte ańo.s Preparadas, pues, con la carencia de las partes aqueas, (como arriba dixe) y con el movimienco, llegaron los calores del Octubre, que en tiempo alguno han fido tan irregulares, y refinaron, y calcinaron del todo eftos atomos; y yà por la agitacion, y fricacion de unos cuerpecillos con otros, por la virtud del movimiento, ( caufa effencial del calor) ò yà porque la vecina esfera del fuego los encendiò, ò quizà cl activo calor del Sol encen- 
didas aparecieron en efte Globo, ò Promontorio, que oy es el aflumpto de nueftro miedo, y curiofidad.

La caufa de la variedad de fus colores, es patente, y clara à qualquiera que aya mirado el Cielo à diverfas horas del dia, y de la noche; pues en las nubes eitàn canfados los ojos de vèr la variedad de fus colores; y efta nace de lo raro, sòlido, ò efpongiofo de la nube, y de la materia de que es formada; y ultimamente, de la radiacion del Sol; pues fegun la difpoficion de fu luz, unas veces nos las propone azules, otras blancas, otras negras, verdes, encarnadas, y de orros diferentes colores. Lo mifmo fucede en el Arco Iris; pues fiendo fu formacion de gotas de agua, como eftas eftèn impregniadas de varios azufres, fales, y nitros iluminados diagonalmente de èl, le dà mas viveza à eftas fales con fu tuz, y con fu fituacion; y eftos fon unos metheoros clarifsimos. Aparecen, pues, los nubarrones negros, quando fu materia es grueffa, terrea, $y$ bien unida, que como no puede penetrar fu sòlidèz la luz del Sol, iluminala por una, y otra parte, y falo aparece el bulto terreo, y sòlido. Aparecen azules, quando eftàn las nubes cargadas de mucho nitro, y quando han chupado porciones de los minerales. Aparecen pagizas, quando eftàn raramente impregnaḑas del azufre. Aparecen encarna- 
das, qüando eftàn compueftas de betūnes, $y$ fales promptos à encenderfe, y raros: y efto lo ve mos todoslos dias, $y$ à todas horas; pero nos quedamos necios para toda la vída, por no detener un poco el difcurfo en el examen de eftas criaturas; $y$ folo quando fe nos opone à nueftra vifta algun objero defconocido, nos affufta, y amedrenta como à los nińos la Marimanta ; y el arbitrio que tomamos para falir de confufiones, es preguntar à los Legos, $y$ à las Beatas, què ferà efto ? Y regularmente refponden, que fon nueftros pecados. $\mathrm{Mi}-$ re V.md. fi Dios avia de poner en las nubes una cofa tan mala. En el infierno es de fee que los caftiga, pero en las nubes, no lo he oido decir fino à ellas. La refpuefta mas general, es: Dios lo quiere afsi, afsilo difpone fu Mageftad. Y fi yo de mas joven huviera fabido efta Filofia breve, me avia de aver dedicado à cofer albardas, que es eftudio mas difit: cultofo.

He oido decir à algunas gentes que vieron unas coronas: otros que obfervaron unas piramides falomonicas : otros que era una Cruz de notable grandeza: todo es pofsible, $y$ monftruos mayores fe forman en efta esfera, pues fe han vifto exercitos de hombres armados, caftillos de fuego, ferpienres, toros, y quanta efpecie de animales regiftramos en la tierra. Todas eftas figuras las finge la diftan- 
cia, y la luz de los Aftros, que haciendo reflexion en las nubes, las hace aparecer en effos varios, y monftruofos cuerpos, que por unas partes fon muy denfos, por otras raros, y por otras dèbiles; y ultimamente, defiguales en la materia, y corporatura. La fuma diftancia que ay defde nueftros ojos à aquellos bultos, hace tambien que nos parezcan hombres, y animales, lo que folo es una deforme, y confufa figura, como le fucede al que camina al tiempo que la noche và borrando con fu tiniebla las formas de los cuerpos; pues el cartafco mas meńique, le parece un erguido gigante; $y$ cree por entonces, que fe vie-: ne acercando à èl con una lanza, y otras femejantes vifiones que pinta la diftancia, y el miedo, que por vulgares, y conocidas de todos no las refiero. Efto es lo que puedo informar à V. md. de la generacion de efte Phenomeno; fi no le fatisface mi doctrina, confulte V.md. 2̀ los Phyficos Mathematicos, que honran effa Corte, ò à los que andan nadando por los Mares, que mas obligacion tienen à faber eftas caufas que yo, pues le comen al Rey mas fueldos que los Capitanes Generales, y à efta hora no hemos logrado que nos impriman media hoja de papel, ni de eftas novedades, ni de los affumptos viejos que contiene qualquiera de las treinta y dos Ciencias 
Mathematicas. Lea V.md. aora el Pronoftico dè efta aparicion, ferè breve, porque no murmure de impertinente el eltilo de mi carta.

\section{PRONOSTICO, O JUICIO \\ DE L PHENOMENO.}

L corazon del hombre (amigo mio, y feñor) como principio del movimiento, es la af-: fiftencia de la virtud vital ; y afsi, los movimienros, y reboluciones de los Cielos, fon la vida de los hombres, y de todo quanto tiene fubfiftencia debaxo del concabo de la Luna. Si los Cielos no fe movieffen, ceffaria la generacion, y corrupcion; y por configuiente todos los movimientos naturales. Imprimen los Aftros las primeras qualidades en el ayre, $y$ de fus temperamentos fe figuen las alteraciones, y mudanzas en todo lo elementado, y fu influencia ; no folo fe incorpora en lo engendrado, y producido, fino es tambien en las entrańas de la tierra, de donde chupa aquellos hus. mos, de que fe congelan los metales; y en la fuperficie fomenta, $y$ anima las producciones de mieffes, y plantas, y dà aliento à todo viviente, fin que, aya cofa que no comprehenda fu dominio, menos el libre alvedrio del hombre, que à efte no le mandan, ni los Aftros, ni los Angeles, 
nilos demonios. Es vifible efte concurfo de las caulas fuperiores, en eftas fublunares; y afsi, quando fon benevolos eftos influxos, gozamos ( con el favor de fu virtud) buena condicion en los humores; y fi es contrario, bebemos en el ayre que refpiramos la inala qualidad del temperamento, afsi los hombres, como brutos, plantas, y todo lo fenfible, ò inanimado. Quando las feñales naturales fon tan evidences como un Eclypfe, un Cometa, ù otro Phenomeno, tiene obligacion el que profefla el eftudio de los Aftros à manifeftar las inclinaciones de fus influxos, fin exceder en las predicciones, los terminos de la prudencia: y fupueftos eftos principios generales, digo, que aviendo fucedido la aparicion de efte Globo en los fignos del Cancer, y el Leon, y parte de la conftelacion de Virgo, es configuiente que caufe notable eftèrilidad en lo criado; porque fon de fu naturaleza eftos fignos fecos, calidos, remiffos, y nebulofos. La imagen de Virgo, es ventofa en toda la parte Boreal, y en la Auftral es témplada; pero no es tanta fu remplanza, que pueda corregir el inmoderado calor de los dos fignos. Jupicer fe hallaba à la hora que obfervè elte Phenomeno, en el figno de Virgo; y elte Planeta miraba folo favorable à la tierra, corrigiendo con fu amigable natural las colericas imprefsio- 
nes de los dos fignos. Efta configuracion, y la de los demàs Planetas, (que omito por no fer neceffaria ) prometen por lo general, fequedad en el ayre, la qual confume las fuentes, y manantiales de los rios: corrompe las aguas: agita vientos calidos, y peftilentes, tempeftades, truenos, horribles rayos; y por la conmixtion de los vientos procelofos, caufarà fubitos naufragios, con pèrdida de Naves. Es deftruccion de los animales utiles al fuitento, $y$ fervicio del hombre, y folo engendra langoitas, lagartos, y otros animalejos podridos, y venenofos. Arruina lo vegerable, corrompe los frutos, y en las mifmas troxes pudre las femillas. De aqui fe figue, que las enfermedades feràn fiebres agudas, morbos colericos, corrupcion en la fangre, efpecialmente à los que eftàn en la edad viril. Promete ruinas de edificios, incendios, hurtos, y depopulaciones. En lo Politico, mueve duras guerras, tumultos, fediciones, iras, y violencias de los Principes : de que fe feguiràn injurias, deftierros, Carceles, y defeftimacion de los virtuofos. Mueve, ultimamente, el apetito à las cofas ilicitas, y à la tranfgrefsion de las Leyes. Eftos efectos promete la vifion de efte Phenomeno: los quales todos moderarà, aunque poco, la buena influencia del Jupiter. 
En la duracion de los efectos de eftas caufas, como fon Eclyples, Cometas, y nuevos Phenomenos, nunca lían variado los Aftrologos, porque todos uniformes afirman, que quantas horas durare el Eclypfe, Cometa, o Phenomeno, otros tantos anos duraràn fus influxos: $y$ aviendo durado efte, por mi obfervacion, quatro horas, otros tantos feràn los ańos que hemos de padecer fus deftemplanzas ; falvo que no aparecerà otro nuevo de contraria condicion, que los deftruya, defalojando del ayre fus materias, evaporandolas, ò confumiendolas. A lostres mefes defpues de la aparicion, fuelen comenzar los efectos à caufar la ruina en la tierra, aunque muchos Aftrologos obfervadores han manifeftado, que los infuxos, ò foplos de eftos Phemenos, comienzan antes̄ de aparecer lentamente; y defpues de aparecidos, con mas fuerza : y yo me arrimo à efte fentir, afsi por fu actividad, como por la cercania de la region adonde fe hacen vifibles.

\section{PREVENCIONES PARA HUIR LA MALA. condicion de los infuxos del Pbenomeno.}

Lultimo mandato que contiene la que re1 cibi de V.md.es pedirme recetas con que 
Librarfe del rigor que amenazan à los cuerpos eftos irregulares influxos, ò atomos, que forbidos en el mifno inftante que refpiramos, inficionan las partes internas de nueftra organizacion. El argumento es dificultofo; porque fiendo la caufa general, y dañando efta, no folo à nueftros cuerpos, fino tambien los frutos, y animales, que brota la naturaleza para alimenes tarlos, es quafi impofsible corregir, y atender à un tiempo à la vigilancia de tantos enemigos. No obftante, V.md. ha de procurat primeramente alegrar el animo, de cal fuerte, que no le dexe cuidado ruidolo à que arender : la conciencia, ante todas colas, mala, ha de limpiar V.md. con tal cuidado, como fi huvieffe de moxir oy à los flechazos de eftos influxos; y aguardar con rifa chriftiana qualquiera trabajo, o enfermedad, ò affalto, que no ay cofa mas facil, que conformate con lo que no tiene remedio. V.md. ha de hacer moderado exercio ; porque del mucho, confeguirà el anunciado encendimiento de la fangre, y turbado movimiento en efte liquido; y efte le ha de elegir à unas horas acomodadas: y en las Eftaciones de Invierno, y Primavera, de Sol à Sol, que en eftas horas eftà mas limpio el ayre: y en el Eftio, y Orońo, por las mañanas, y nunca por las noches. La habicacion 
en que V.md: ha de afsiftir, ha de procurar limpiarla, afsi de la hediondèz del ayre domeftico, como de la impureza del viento: y efto lo lograrà, regandola con el vinagre rofado, ò con las flores olorofas del Mayo ; y en el Invierno, ufar de algunas lumbres aromaticas, cuya defcripcion, y compoficion, hallarà V.md. en mi librito de la Vida Natural, y Carholica. Deordinario llevarà V.md. configo algunos ajos crudos, y los mazcarà alguna vez. En el ufo del vino, es neceffario que V.md. fe acorte la racion; porque ademàs de que eftos años ferà de mala naturaleza, es por si dańofo, aùn el mas dulce, $y$ mas bien circunftanciado. La mayor copia de enfermedades, feràn inflamaciones internas : beba V.md. algunas orchatas de las quatro fimientes, y leche de cabras, en ayunas, que efto le ferà,afsi à la de V.md.como à qualquiera de las natiralezas viriles, mas amigable; y como el eftomago no fe enoje, bebala V.md. aunquie fea todo el Verano, y Eftio. Las carnes que V.molaya de comer, fean frefcas, y nada menos que ufar de las faladas, ni de las legunibres de mala cafta, como fon los bretones, remolachas, berzas, zanahorias, ni repollos: fon mas del cafo, y mas benignas las lechugas, ef carolas, y efparragos. Las purgas, opiaras, vomitivos, y otros julepes, y jaraves, huya V.md. 
de ellos como de la muerte y fin gravifsima caufa, no fe decermine à beberlas, porque atceraràn demafiado los cuerpos; y eftando eftos preparados, à la inflamacion interna la excitan, y la ayudan; y apoderada una vez de los miembros principales, es dificultofifsima de apagar. Las fangtias pueden fer mas feguras ; pero, eftas fe han de decerminar fegun el tiempo, ta edad, complexion, y naturaleza del mal. Yo efpero en Dios, que V. md. ha de lograr falud, y no llegas rà el cafo de que montanteen los Medicos con las fangrias, purgas, y ventofas; pero fieftellegare, avife V.mid. que como yo halle en mis fuerzas, en mi oftudio, ò en mi afsiftencia, el valar, y buena eleccinn, que tengo en mi vos lunead para fervirlo, V.md. quedarà fano de la mayor enfermedad, y libre del mas proximo peligro.

Suplico à V.md. que otro dia me dè mas tiempo, para confultar con mist talencos eftos encargos; pues fon breves, las horas que permire un Correo, para cumplir empeños do efta conk fideracion. Por Cartas avifarèyo à Vimd. de las circunftancias que ocurran; y V.md. pevdone, que aya eftado tan libre, con el refpeto que le debo, en las primeras hojas, que yo confieffo que me alterò un poco el mandato, por ol hor? ror con que uniro à eltas mąrevias, tan aborteci- 
das de la vulgaridad, y por la precilsion de la refipuefta à buelta de Correo. No le doy à V.md. mas padtinos, que aver executado fu precepto ; y aunque alguna vez regańe, porque lo rabiofo de mi genio, me muerde las confideraciones : no haga V.md. cafo de effo, que yo bien conozco en $\mathrm{mi}_{\text {, }}$ que he de hacer quanto V.mind. me mandare, fea lo que fuere : aora viva V.md. feliz, y logre larga vida, coronada de felicidades, en la dichofa compañia de cfla Señora, à cuyos pies quedo con toda veneracion. Oy eftamos à 14 . de Noviembre de 1730 .

\section{B. L.M. de V.md. fu fervidor, y buen amigo,}

Diego de Torres.

Señor Don Juan Ventura, mi amigo.

IDonde efte, fe hallarà el Papel nuevo, intitulado: Vida Natural, $y$ Catbolica, Medicina fegura para mantener menos enferma la organizacion del cuerpo, y affegurar ai alma la eterna falud, fu Autor Don Diego de Torres, Cachedratico de Mathematicas en la Univerfidad de Salamanca. 
es

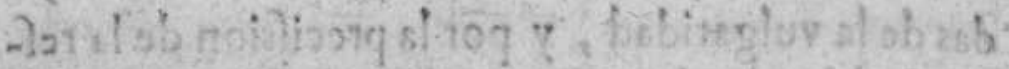

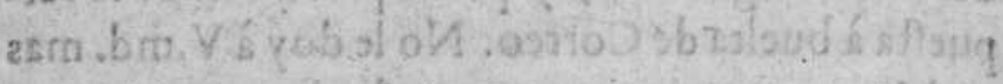

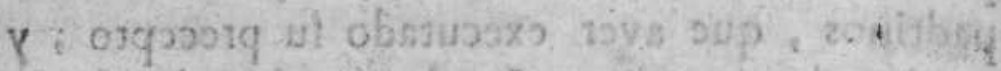

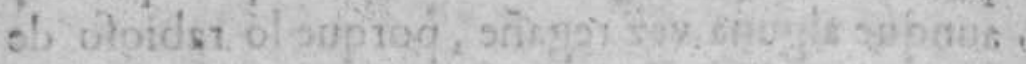

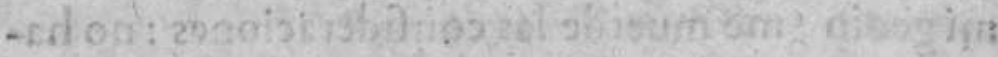

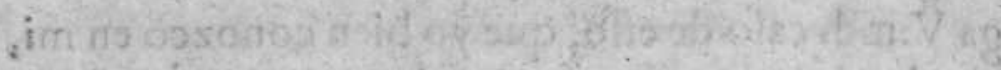

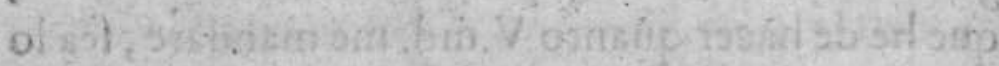

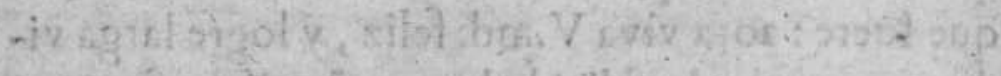

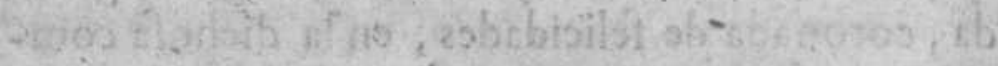

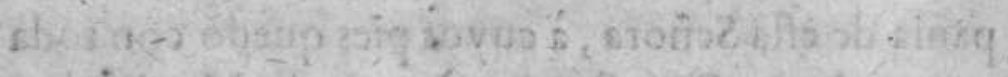

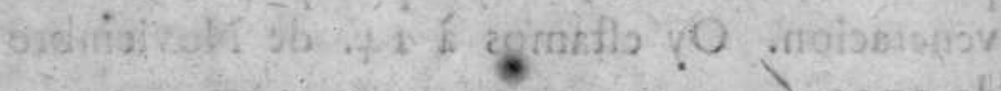

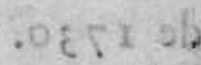

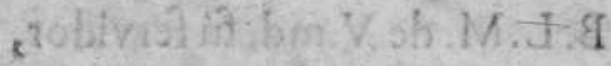

$$
\begin{aligned}
& \text { cightas noud? }
\end{aligned}
$$

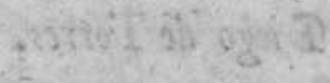

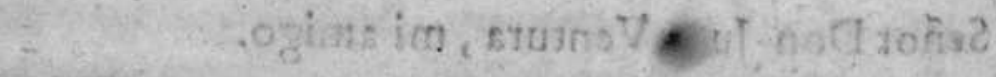
c

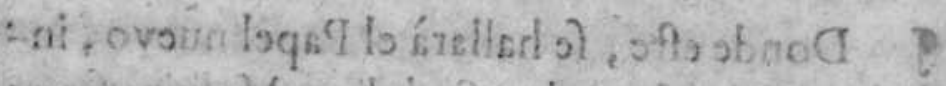

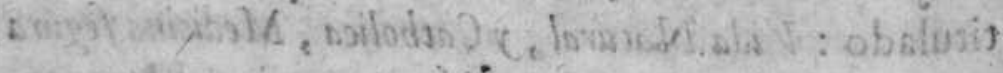

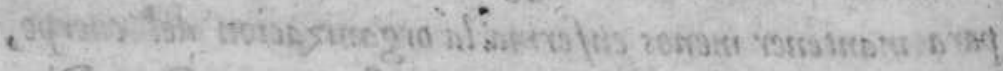
-

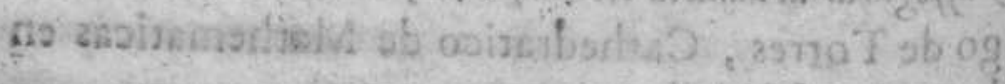

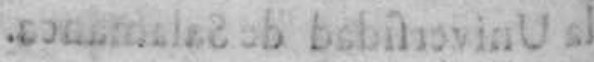






$$
\begin{aligned}
& 34 \text { pur } \\
& \text { M. SIE pero } 1730
\end{aligned}
$$



